\title{
The Initial Boundary Value Problem for Modified Zakharov System
}

\author{
Lang Li, Shaomei Fang* \\ Department of Mathematics, South China Agricultural University, Guangzhou, China \\ Email: ${ }^{*}$ dz90@scau.edu.cn
}

Received 6 July 2014; accepted 10 April 2015; published 16 April 2015

Copyright (C) 2015 by authors and Scientific Research Publishing Inc.

This work is licensed under the Creative Commons Attribution International License (CC BY). http://creativecommons.org/licenses/by/4.0/

c) (i) Open Access

\section{Abstract}

In this paper, we consider the initial boudary value problem for modified Zakharov system in 3 dimensions with small initial condition. By using the continuity lemma and the linear interpolation theory, together with the properties of Sobolev spaces and the Galerkin method, we obtain the existence and uniqueness of the global solution.

\section{Keywords}

\section{Modified Zakharov System, Galerkin Method, A Prior Estimate}

\section{Introduction}

In this paper, we study the global existence and uniqueness of solutions for a modified Zakharov system with initial boundary value conditions as follows.

$$
\begin{aligned}
& i E_{t}+\Delta E-H^{2} \Delta^{2} E-n E=0, \\
& n_{t t}-\Delta n+H^{2} \Delta^{2} n-\Delta|E|^{2}=0, \\
& \left.E\right|_{t=0}=E_{0}(x),\left.n\right|_{t=0}=n_{0}(x),\left.\quad n_{t}\right|_{t=0}=n_{1}(x), x \in \Omega \subset \mathbb{R}^{3}, \\
& \left.E\right|_{\partial \Omega}=\left.\frac{\partial E}{\partial v}\right|_{\partial \Omega}=\left.\frac{\partial(\nabla E)}{\partial v}\right|_{\partial \Omega}=0, t \geq 0, \\
& \left.n\right|_{\partial \Omega}=0,\left.\quad \frac{\partial(\nabla n)}{\partial v}\right|_{\partial \Omega}=0,
\end{aligned}
$$

${ }^{*}$ Corresponding author. 
where $E: \mathbb{R}^{3+1} \rightarrow \mathbb{C}^{3}$ represents the slowly varying envelope of the electric field, $n: \mathbb{R}^{3+1} \rightarrow \mathbb{R}$ denotes deviation of the ion density from its mean, and $H$ is the dimensionless quantum parameter given by the ratio of the ion plasmon and electron thermal energies.

The classical Zakharov system was derived by Zakharov to describe the propagation of Langmuir waves in a plasma [1]. In the past decade, a large amount of work has been devoted to the existence problem for the classical Zakharov system. For instance, the Fourier restriction norm method, applies to this problem, and under appropriate assumption on the data, several existence results have been established. The local well-posedness of this problem on $\mathbb{R}^{2}$ in the energy space $H^{1} \times L^{2} \times \hat{H}^{-1}$ was first obtained by Bourgain and Colliander [2], which was improved to $H^{1 / 2} \times L^{2} \times H^{-1}$ by Ginibre, Tsutsumi and Velo [3]. Recently, Bejenaru, Herr, Holmer and Tataru have obtained local well-posedness in $L^{2} \times H^{-1 / 2} \times H^{-3 / 2}$ [4]. Another approach was initiated by Colliander, Keel, Stafflani, Takaoka, and Tao in [5], called the I-method. This method was successfully applied by these authors to the Kdv and modified Kdv equation. In [6], this method was also used to construct global solutions for the one dimensional Zakharov system.

However, some important effects, such as quantum effects, have been ignored in the classical Zakharov system. The importance of quantum effects in ultrasmall electronic devices, dense astrophysical plasma systems and laser plasmas has produced an increasing interest on the investigation of the quantum counterpart of some of plasma physics phenomena [7]. By using a quantum fluid approach, Garcia has obtained the following modified Zakharov system [8]:

$$
\begin{aligned}
& i E_{t}+E_{x x}-H^{2} E_{x x x x}-n E=0, \\
& n_{t t}-n_{x x}+H^{2} n_{x x x x}-|E|_{x x}^{2}=0 .
\end{aligned}
$$

Recently, S. You and B. Guo have considered the existence and uniqueness of the global solution to the initial boundary value problem for the above system in 1 dimension and 2 dimensions, respectively [9] [10]. Motivated by their result, we consider the global existence of solutions in 3 dimensions with small initial condition. As is standard, the problem is to obtain a prior estimate of higher derivatives of solutions in some suitable function spaces. To overcome higher dimensional difficulty, we use continuity lemma and the linear interpolation theory, together with the properties of Sobolev spaces to handle it.

Now we give some notations:

-For $1 \leq q \leq \infty$, we denote by $L^{q}(\Omega)$ or simply $L^{q}$, the space of all $q$ times integrable functions in $\Omega$ equipped with norm $\|\cdot\|_{L^{q}(\Omega)}$ or simply $\|\cdot\|_{L^{q}}$.

$$
\|f\|_{L^{p}(\Omega)}=\left(\iint_{\Omega}|f|^{p} \mathrm{~d} x\right)^{1 / p},(1 \leq p<\infty),\|f\|_{L^{\infty}(\Omega)}=\operatorname{ess} \sup (|f(x)|: x \in \Omega),(p=\infty) .
$$

-Let $H^{s}(\Omega)$ be the usual Sobolev space of complex-valued functions with the norm

$$
\|f\|_{H^{s}(\Omega)}=\left(\sum_{|\alpha| \leq s} \int\left|D_{\Omega}^{\alpha} f\right|^{2} \mathrm{~d} x\right)^{1 / 2} .
$$

-We denote by $C$ a positive constant which may change from one line to the next line.

\section{A Prior Estimates}

To study the smooth solution of the modified Zakharov system, we introduce function $\varphi$, and transform Equations (1.1)-(1.5) into the form:

with initial condition

$$
\begin{aligned}
& i E_{t}+\Delta E-H^{2} \Delta^{2} E-n E=0, \\
& n_{t}-\Delta \varphi=0 \\
& \varphi_{t}-n+H^{2} \Delta n-|E|^{2}=0,
\end{aligned}
$$

$$
\left.E\right|_{t=0}=E_{0}(x),\left.n\right|_{t=0}=n_{0}(x),\left.\varphi\right|_{t=0}=\varphi_{0}(x), x \in \Omega
$$

and boundary condition 


$$
\begin{aligned}
& \left.E\right|_{\partial \Omega}=\left.\frac{\partial E}{\partial v}\right|_{\partial \Omega}=\left.\frac{\partial(\nabla E)}{\partial v}\right|_{\partial \Omega}=0, \\
& \left.n\right|_{\partial \Omega}=0,\left.\frac{\partial(\nabla n)}{\partial v}\right|_{\partial \Omega}=0 .
\end{aligned}
$$

Lemma 2.1. Assume that $E_{0} \in L^{2}$, and $(E, n, \varphi)$ is the solution to (2.1)-(2.6), then we have

$$
\|E(t)\|_{L^{2}}^{2}=\left\|E_{0}\right\|_{L^{2}}^{2} \text {. }
$$

Proof. Multiplying Equation (2.1) by $\bar{E}$, and integrating over $\Omega$, we have

$$
\left(i E_{t}+\Delta E-H^{2} \Delta^{2} E-n E, E\right)=0 .
$$

Since

$$
\begin{gathered}
\operatorname{Im}\left(i E_{t}, E\right)=\frac{1}{2} \frac{\mathrm{d}}{\mathrm{d} t}\|E\|_{L^{2}}^{2}, \quad \operatorname{Re} \int_{\mathbb{R}^{3}} \Delta E \cdot \bar{E} \mathrm{~d} x=-\|\nabla E\|_{L^{2}}^{2}, \\
\int_{\mathbb{R}^{3}} H^{2} \Delta^{2} E \cdot \bar{E} \mathrm{~d} x=H^{2}\|\Delta E\|_{L^{2}}^{2}, \quad \int_{\mathbb{R}^{3}} n E \cdot \bar{E} \mathrm{~d} x=\int_{\mathbb{R}^{3}} n|E|^{2} \mathrm{~d} x .
\end{gathered}
$$

Taking the imaginary part of Equation (2.7), then we have

$$
\frac{1}{2} \frac{\mathrm{d}}{\mathrm{d} t}\|E\|_{L^{2}}^{2}=0 .
$$

Lemma 2.2. [11] (Continuity lemma) Assume that $f(t)$ is a nonnegative continuous function defined on $\mathbb{R}^{+}$and

$$
f(t) \leq a+b f^{k}(t), a, b>0, k>1 .
$$

If $a^{k-1} b<\frac{(k-1)^{k-1}}{k^{k}}$ and $f(0) \leq a$ also hold, then $f(t)$ is bounded in $\mathbb{R}^{+}$.

Lemma 2.3. [11] (Gargliardo-Nirenberg inequality) Assume that $u \in L^{q}(\Omega), D^{m} u \in L^{r}(\Omega), \quad \Omega \subset \mathbb{R}^{n}$, $1 \leq q, \quad r \leq \infty, 0 \leq j \leq m$, then

$$
\left\|D^{j} u\right\|_{L^{p}} \leq C\left\|D^{m} u\right\|_{L^{r}}^{\alpha}\|u\|_{L^{q}}^{1-\alpha},
$$

where $0 \leq \frac{j}{m} \leq \alpha \leq 1, \frac{1}{p}=\frac{j}{n}+\alpha\left(\frac{1}{r}-\frac{m}{n}\right)+(1-\alpha) \frac{1}{q}$.

Lemma 2.4. Assume that $(E, n, \varphi)$ is the solution to (2.1)-(2.6), and $E_{0}(x) \in H_{0}^{2}, n_{0} \in H_{0}^{1}, \varphi_{0} \in H_{0}^{1}$, then we have

$$
\sup _{0 \leq t \leq T}\left[\|E\|_{H_{0}^{2}}^{2}+\|n\|_{H_{0}^{1}}^{2}+\|\varphi\|_{H_{0}^{1}}^{2}\right] \leq C .
$$

Proof. Multiplying Equation (2.1) by $\bar{E}_{t}$, and integrating over $\Omega$, we have

$$
\left(i E_{t}+\Delta E-H^{2} \Delta^{2} E-n E, E_{t}\right)=0 .
$$

Since

$$
\begin{aligned}
& \left(i E_{t}, E_{t}\right)=i\left\|E_{t}\right\|_{L^{2}}^{2}, \quad \int_{\mathbb{R}^{3}} \Delta E \cdot \bar{E}_{t} \mathrm{~d} x=-\frac{1}{2} \frac{\mathrm{d}}{\mathrm{d} t}\|\nabla E\|_{L^{2}}^{2}, \\
& \int_{\mathbb{R}^{3}} H^{2} \Delta^{2} E \cdot \bar{E}_{t} \mathrm{~d} x=H^{2} \frac{1}{2} \frac{\mathrm{d}}{\mathrm{d} t}\|\Delta E\|_{L^{2}}^{2}, \quad \int_{\mathbb{R}^{3}} n E \cdot \bar{E}_{t} \mathrm{~d} x=\frac{1}{2} \int_{\mathbb{R}^{3}} n|E|_{t}^{2} \mathrm{~d} x,
\end{aligned}
$$

then taking the real part of Equation (2.8), we have 


$$
\frac{\mathrm{d}}{\mathrm{d} t}\left(\|\nabla E\|_{L^{2}}^{2}+H^{2}\|\Delta E\|_{L^{2}}^{2}+\int_{\mathbb{R}^{3}} n|E|^{2} \mathrm{~d} x\right)-\int_{\mathbb{R}^{3}} n_{t}|E|^{2} \mathrm{~d} x=0,
$$

where we have used the fact

$$
\frac{\mathrm{d}}{\mathrm{d} t} \int_{\mathbb{R}^{3}} n|E|^{2} \mathrm{~d} x=\int_{\mathbb{R}^{3}} n_{t}|E|^{2} \mathrm{~d} x+\int_{\mathbb{R}^{3}} n|E|_{t}^{2} \mathrm{~d} x .
$$

Similarly, multiplying Equation (2.2) by $n$, and integrating over $\Omega$, we have

$$
\left(n_{t}-\Delta \varphi, n\right)=0 \text {, }
$$

i.e.

$$
\begin{aligned}
\left(n_{t}-\Delta \varphi, n\right) & =\left(n_{t}, n\right)-\left(\Delta \varphi, \varphi_{t}+H^{2} \Delta n-|E|^{2}\right) \\
& =\frac{1}{2} \frac{\mathrm{d}}{\mathrm{d} t}\|n\|_{L^{2}}^{2}+\frac{1}{2} \frac{\mathrm{d}}{\mathrm{d} t}\|\nabla \varphi\|_{L^{2}}^{2}-\left(\Delta \varphi, H^{2} \Delta n-|E|^{2}\right) \\
& =\frac{1}{2} \frac{\mathrm{d}}{\mathrm{d} t}\|n\|_{L^{2}}^{2}+\frac{1}{2} \frac{\mathrm{d}}{\mathrm{d} t}\|\nabla \varphi\|_{L^{2}}^{2}-\left(n_{t}, H^{2} \Delta n-|E|^{2}\right) \\
& =\frac{1}{2} \frac{\mathrm{d}}{\mathrm{d} t}\|n\|_{L^{2}}^{2}+\frac{1}{2} \frac{\mathrm{d}}{\mathrm{d} t}\|\nabla \varphi\|_{L^{2}}^{2}+\frac{H^{2}}{2} \frac{\mathrm{d}}{\mathrm{d} t}\|\nabla n\|_{L^{2}}^{2}+\int_{\mathbb{R}^{3}} n_{t}|E|^{2} \mathrm{~d} x=0 .
\end{aligned}
$$

Adding Equation (2.9) to Equation (2.11), we deduce

$$
\frac{\mathrm{d}}{\mathrm{d} t}\left(\|\nabla E\|_{L^{2}}^{2}+H^{2}\|\Delta E\|_{L^{2}}^{2}+\int_{\mathbb{R}^{3}} n|E|^{2} \mathrm{~d} x+\frac{1}{2}\|n\|_{L^{2}}^{2}+\frac{1}{2}\|\nabla \varphi\|_{L^{2}}^{2}+\frac{H^{2}}{2}\|\nabla n\|_{L^{2}}^{2}\right)=0 .
$$

Set $\Psi(t)=\|\nabla E\|_{L^{2}}^{2}+H^{2}\|\Delta E\|_{L^{2}}^{2}+\int_{\mathbb{R}^{3}} n|E|^{2} \mathrm{~d} x+\frac{1}{2}\|n\|_{L^{2}}^{2}+\frac{1}{2}\|\nabla \varphi\|_{L^{2}}^{2}+\frac{H^{2}}{2}\|\nabla n\|_{L^{2}}^{2}$, then we have

$$
\Psi(t)=\Psi(0),
$$

therefore, using Young's inequality, we have

$$
\begin{aligned}
& \|\nabla E\|_{L^{2}}^{2}+H^{2}\|\Delta E\|_{L^{2}}^{2}+\frac{1}{2}\|n\|_{L^{2}}^{2}+\frac{1}{2}\|\nabla \varphi\|_{L^{2}}^{2}+\frac{H^{2}}{2}\|\nabla n\|_{L^{2}}^{2} \\
& \leq \Psi(0)+\left.\left|\int_{\mathbb{R}^{3}} n\right| E\right|^{2} \mathrm{~d} x \mid \leq \Psi(0)+\frac{\varepsilon}{4}\|n\|_{L^{2}}^{2}+\frac{1}{\varepsilon}\|E\|_{L^{4}}^{4} .
\end{aligned}
$$

Choosing $\varepsilon$ such that $\frac{\varepsilon}{4} \leq \frac{1}{2}$, and by Lemma 2.3, we have

$$
\|\nabla E\|_{L^{2}}^{2} \leq \Psi(0)+\frac{1}{\varepsilon} C\|E\|_{L^{4}}^{4} \leq \Psi(0)+\frac{1}{\varepsilon} C\|E\|_{L^{2}}\|\nabla E\|_{L^{2}}^{3} .
$$

Set $m=\|\nabla E\|_{L^{2}}^{2}$, then the above inequality can be simplified as $m \leq a+b m^{\frac{3}{2}}$, where $a=\Psi(0), b=\frac{1}{\varepsilon} C\left\|E_{0}\right\|_{L^{2}}$, then using Lemma 2.2 yields

$$
\|\nabla E\|_{L^{2}}^{2} \leq C
$$

if the initial condition is small enough. Substituting it into Equation (2.12), we have

$$
\|\nabla E\|_{L^{2}}^{2}+H^{2}\|\Delta E\|_{L^{2}}^{2}+\|n\|_{L^{2}}^{2}+\|\nabla \varphi\|_{L^{2}}^{2}+\|\nabla n\|_{L^{2}}^{2} \leq C .
$$

Lemma 2.5. Assume that $(E, n, \varphi)$ is the solution to (2.1)-(2.6), and $E_{0}(x) \in H_{0}^{4}, n_{0} \in H_{0}^{2}, \varphi_{0} \in H_{0}^{2}$, we 
have

$$
\sup _{0 \leq t \leq T}\left[\left\|\Delta^{2} E\right\|_{L^{2}}^{2}+\|n\|_{H^{2}}^{2}+\left\|n_{t}\right\|_{L^{2}}^{2}+\|\Delta \varphi\|_{L^{2}}^{2}+\left\|E_{t}\right\|_{L^{2}}^{2}+\left\|\varphi_{t}\right\|_{L^{2}}^{2}\right] \leq C .
$$

Proof. Differentiating Equation (2.1) with respect to $t$, and then multiplying it by $\bar{E}_{t}$, integrating over $\Omega$, we have

$$
\left(i E_{t t}+\Delta E_{t}-H^{2} \Delta^{2} E_{t}-n_{t} E-n E_{t}, E_{t}\right)=0 .
$$

Since

$$
\begin{aligned}
& \operatorname{Im}\left(i E_{t t}, E_{t}\right)=\frac{1}{2} \frac{\mathrm{d}}{\mathrm{d} t}\left\|E_{t}\right\|_{L^{2}}^{2}, \quad \int_{\mathbb{R}^{3}} \Delta E_{t} \cdot \bar{E}_{t} \mathrm{~d} x=-\left\|\nabla E_{t}\right\|_{L^{2}}^{2}, \\
& \int_{\mathbb{R}^{3}} H^{2} \Delta^{2} E_{t} \cdot \bar{E}_{t} \mathrm{~d} x=H^{2}\left\|\Delta E_{t}\right\|_{L^{2}}^{2}, \quad \int_{\mathbb{R}^{3}} n_{t} E \cdot \bar{E}_{t} \mathrm{~d} x=\frac{1}{2} \int_{\mathbb{R}^{3}} n_{t}|E|_{t}^{2} \mathrm{~d} x .
\end{aligned}
$$

Taking the imaginary part of Equation (2.13) yields

$$
\frac{1}{2} \frac{\mathrm{d}}{\mathrm{d} t}\left\|E_{t}\right\|_{L^{2}}^{2}-\operatorname{Im}\left(n_{t} E, E_{t}\right)=0,
$$

therefore, using Hölder's inequality and Sobolev imbedding, we have

$$
\frac{\mathrm{d}}{\mathrm{d} t}\left\|E_{t}\right\|_{L^{2}}^{2} \leq C\left\|n_{t}\right\|_{L^{2}}\|E\|_{L^{\infty}}\left\|E_{t}\right\|_{L^{2}} \leq C\left(\left\|E_{t}\right\|_{L^{2}}^{2}+\left\|n_{t}\right\|_{L^{2}}^{2}\right) .
$$

Differentiating Equation (2.2) with respect to $t$, and then multiplying it by $n_{t}$, integrating over $\Omega$, we have

$$
\left(n_{t t}-\Delta \varphi_{t}, n_{t}\right)=0 \text {, }
$$

i.e.

$$
\left(n_{t t}, n_{t}\right)+\left(-\Delta n+H^{2} \Delta^{2} n-\Delta|E|^{2}, n_{t}\right)=0,
$$

therefore

$$
\begin{aligned}
& \frac{\mathrm{d}}{\mathrm{d} t}\left(\left\|n_{t}\right\|_{L^{2}}^{2}+\|\nabla n\|_{L^{2}}^{2}+H^{2}\|\Delta n\|_{L^{2}}^{2}\right) \\
& \leq 2\left|\left(\Delta|E|^{2}, n_{t}\right)\right| \leq C\left(\left\|n_{t}\right\|_{L^{2}}^{2}+\|E\|_{L^{\infty}}^{2}\|\Delta E\|_{L^{2}}^{2}+\|\nabla E\|_{L^{4}}^{4}\right) \leq C\left(\left\|n_{t}\right\|_{L^{2}}^{2}+1\right) .
\end{aligned}
$$

Adding Equation (2.14) to Equation (2.15), we have

$$
\frac{\mathrm{d}}{\mathrm{d} t}\left(\left\|E_{t}\right\|_{L^{2}}^{2}+\left\|n_{t}\right\|_{L^{2}}^{2}+\|\nabla n\|_{L^{2}}^{2}+H^{2}\|\Delta n\|_{L^{2}}^{2}\right) \leq C\left(\left\|E_{t}\right\|_{L^{2}}^{2}+\left\|n_{t}\right\|_{L^{2}}^{2}+1\right) .
$$

Using Gronwall's inequality, we have

$$
\left\|E_{t}\right\|_{L^{2}}^{2}+\left\|n_{t}\right\|_{L^{2}}^{2}+\|\nabla n\|_{L^{2}}^{2}+\|\Delta n\|_{L^{2}}^{2} \leq C .
$$

From Equation (2.1), Equation (2.2), and Equation (2.3), it easily get

$$
\left\|\Delta^{2} E\right\|_{L^{2}}^{2} \leq C,\|\Delta \varphi\|_{L^{2}}^{2} \leq C,\left\|\varphi_{t}\right\|_{L^{2}}^{2} \leq C .
$$

\section{Existence and Uniqueness of Global Solution}

Theorem 3.1. Assume that $E_{0}(x) \in H_{0}^{4}, n_{0} \in H_{0}^{2}, \varphi_{0} \in H_{0}^{2}$, then there exists a unique global solution of the initial boundary value problem (2.1)-(2.6),

$$
\begin{aligned}
& \Delta^{2} E(t, x) \in L^{\infty}\left(0, T ; L^{2}\right), \quad E_{t}(t, x) \in L^{\infty}\left(0, T ; L^{2}\right), \\
& n(t, x) \in L^{\infty}\left(0, T ; H_{0}^{2}\right), \quad n_{t}(t, x) \in L^{\infty}\left(0, T ; L^{2}\right),
\end{aligned}
$$




$$
\Delta \varphi(t, x) \in L^{\infty}\left(0, T ; L^{2}\right), \quad \varphi_{t}(t, x) \in L^{\infty}\left(0, T ; L^{2}\right) .
$$

Proof. We first give the proof of the uniqueness of the solution. Suppose $\left(E_{1}, n_{1}, \varphi_{1}\right)$ and $\left(E_{2}, n_{2}, \varphi_{2}\right)$ are two solutions of (2.1)-(2.6). Let

$$
\tilde{E}=E_{1}-E_{2}, \tilde{n}=n_{1}-n_{2}, \tilde{\varphi}=\varphi_{1}-\varphi_{2},
$$

therefore

$$
\begin{aligned}
& i \tilde{E}_{t}+\Delta \tilde{E}-H^{2} \Delta^{2} \tilde{E}-n_{1} E_{1}+n_{2} E_{2}=0, \\
& \tilde{n}_{t}-\Delta \tilde{\varphi}=0, \\
& \tilde{\varphi}_{t}-\tilde{n}+H^{2} \Delta \tilde{n}-\left|E_{1}\right|^{2}+\left|E_{2}\right|^{2}=0,
\end{aligned}
$$

with initial condition

$$
\left.\tilde{E}\right|_{t=0}=0,\left.\tilde{n}\right|_{t=0}=0,\left.\tilde{\varphi}\right|_{t=0}=0 .
$$

Multiplying Equation (3.1) by $\tilde{E}$, and integrating over $\Omega$, we have

$$
\left(i \tilde{E}_{t}+\Delta \tilde{E}-H^{2} \Delta^{2} \tilde{E}-n_{1} E_{1}+n_{2} E_{2}, \tilde{E}\right)=0
$$

taking the imaginary part yields

therefore

$$
\frac{1}{2} \frac{\mathrm{d}}{\mathrm{d} t}\|\tilde{E}\|_{L^{2}}^{2}+\operatorname{Im}\left(-n_{1} E_{1}+n_{2} E_{2}, \tilde{E}\right)=0
$$

$$
\frac{\mathrm{d}}{\mathrm{d} t}\|\tilde{E}\|_{L^{2}}^{2} \leq C\left(\left\|E_{1}\right\|_{L^{\infty}}\|\tilde{n}\|_{L^{2}}+\left\|n_{2}\right\|_{L^{\infty}}\|\tilde{E}\|_{L^{2}}\right)\|\tilde{E}\|_{L^{2}} \leq C\left(\|\tilde{E}\|_{L^{2}}^{2}+\|\tilde{n}\|_{L^{2}}^{2}\right) .
$$

Multiplying Equation (3.3) by $\tilde{\varphi}$, and integrating over $\Omega$, we have

$$
\left(\tilde{\varphi}_{t}-\tilde{n}+H^{2} \Delta \tilde{n}-\left|E_{1}\right|^{2}+\left|E_{2}\right|^{2}, \tilde{\varphi}\right)=0 .
$$

Since

$$
\begin{aligned}
& \left(\tilde{\varphi}_{t}, \tilde{\varphi}\right)=\frac{1}{2} \frac{\mathrm{d}}{\mathrm{d} t}\|\tilde{\varphi}\|_{L^{2}}^{2}, \quad|(-\tilde{n}, \tilde{\varphi})| \leq C\left(\|\tilde{n}\|_{L^{2}}^{2}+\|\tilde{\varphi}\|_{L^{2}}^{2}\right), \\
& \left(H^{2} \Delta \tilde{n}, \tilde{\varphi}\right)=H^{2}(\tilde{n}, \Delta \tilde{\varphi})=H^{2}\left(\tilde{n}, \tilde{n}_{t}\right)=\frac{H^{2}}{2} \frac{\mathrm{d}}{\mathrm{d} t}\|\tilde{n}\|_{L^{2}}^{2}, \\
& \left|\left(-\left|E_{1}\right|^{2}+\left|E_{2}\right|^{2}, \tilde{\varphi}\right)\right|=\left|\left(\left(E_{1}-E_{2}\right) \bar{E}_{1}+E_{2}\left(\bar{E}_{1}-\bar{E}_{2}\right), \tilde{\varphi}\right)\right| \\
& \leq C\left(\left\|E_{1}\right\|_{L^{\infty}}\|\tilde{E}\|_{L^{2}}+\left\|E_{2}\right\|_{L^{\infty}}\|\tilde{E}\|_{L^{2}}\right)\|\tilde{\varphi}\|_{L^{2}} \\
& \leq C\left(\|\tilde{E}\|_{L^{2}}^{2}+\|\tilde{\varphi}\|_{L^{2}}^{2}\right),
\end{aligned}
$$

therefore

$$
\frac{1}{2} \frac{\mathrm{d}}{\mathrm{d} t}\|\tilde{\varphi}\|_{L^{2}}^{2}+\frac{H^{2}}{2} \frac{\mathrm{d}}{\mathrm{d} t}\|\tilde{n}\|_{L^{2}}^{2} \leq C\left(\|\tilde{n}\|_{L^{2}}^{2}+\|\tilde{\varphi}\|_{L^{2}}^{2}+\|\tilde{E}\|_{L^{2}}^{2}\right)
$$

Adding Equation (3.5) to Equation (3.6), we have

$$
\frac{\mathrm{d}}{\mathrm{d} t}\left(\|\tilde{\varphi}\|_{L^{2}}^{2}+\|\tilde{n}\|_{L^{2}}^{2}+\|\tilde{E}\|_{L^{2}}^{2}\right) \leq C\left(\|\tilde{n}\|_{L^{2}}^{2}+\|\tilde{\varphi}\|_{L^{2}}^{2}+\|\tilde{E}\|_{L^{2}}^{2}\right) .
$$

Using Gronwall's inequality and the initial condition Equation (3.4), we can obtain

$$
\tilde{E} \equiv 0, \quad \tilde{n} \equiv 0, \quad \tilde{\varphi} \equiv 0 .
$$


Next we show the existence of the solution.

By using the Garlerkin method, choose basic functions $\left\{\omega_{j}\right\} \subset H_{0}^{4}$ such that $-\Delta \omega_{j}=\lambda_{j} \omega_{j},(j=1,2, \cdots, v)$, where $\lambda_{j}(j=1,2, \cdots, v)$ are different corresponding eigenvalues. The approximate solution of problem (2.1)(2.6) can be written as

$$
\begin{aligned}
E^{v} & =\sum_{s=1}^{v} \alpha_{s v}(t) \omega_{s}(x), \\
n^{v} & =\sum_{s=1}^{v} \beta_{s v}(t) \omega_{s}(x), \\
\varphi^{v} & =\sum_{s=1}^{v} \gamma_{s v}(t) \omega_{s}(x),
\end{aligned}
$$

where the undetermined coefficients $\alpha_{s v}(t), \beta_{s v}(t), \gamma_{s v}(t)(s=1,2, \cdots, v ; v=1,2, \cdots)$ need to satisfy the following initial value problem of the system of ordinary differential equations

$$
\begin{gathered}
i\left(E_{t}^{v}, \omega_{s}\right)+\left(\Delta E^{v}, \omega_{s}\right)-H^{2}\left(\Delta^{2} E^{v}, \omega_{s}\right)-\left(n^{v} E^{v}, \omega_{s}\right)=0, \\
\left(n_{t}^{v}, \omega_{s}\right)-\left(\Delta \varphi^{v}, \omega_{s}\right)=0, \\
\left(\varphi_{t}^{v}, \omega_{s}\right)-\left(n^{v}, \omega_{s}\right)+H^{2}\left(\Delta n^{v}, \omega_{s}\right)-\left(\left|E^{v}\right|^{2}, \omega_{s}\right)=0,
\end{gathered}
$$

with initial conditions

$$
\begin{aligned}
& \left(E^{v}(0, x), \omega_{s}(x)\right)=\left(E_{0}(x), \omega_{s}(x)\right), \\
& \left(n^{v}(0, x), \omega_{s}(x)\right)=\left(n_{0}(x), \omega_{s}(x)\right), \\
& \left(\varphi^{v}(0, x), \omega_{s}(x)\right)=\left(\varphi_{0}(x), \omega_{s}(x)\right) .
\end{aligned}
$$

According to the basic theory of ordinary differential equations, the above equations have a unique local solution.

Similarly to the proof of Lemma 2.1 and Lemma 2.4, we have

$$
\sup _{0 \leq t \leq T}\left(\left\|E^{v}\right\|_{H_{0}^{1}}+\left\|n^{v}\right\|_{H_{0}^{1}}+\left\|E^{v}{ }_{t}\right\|_{H^{1}}^{2}+\left\|n^{v}{ }_{t}\right\|_{L^{2}}\right) \leq C .
$$

By compactness argument, we can choose subsequences, still denoted by $\left\{E^{v}\right\},\left\{n^{v}\right\}$ such that

$$
\begin{aligned}
& E^{v} \rightarrow E \text { in } L^{\infty}\left(0, T ; H_{0}^{4}\right) \text { weakly star; } \\
& E_{t}^{v} \rightarrow E_{t} \text { in } L^{\infty}\left(0, T ; L^{2}\right) \text { weakly star; } \\
& n^{v} \rightarrow n \text { in } L^{\infty}\left(0, T ; H_{0}^{1}\right) \text { weakly star; } \\
& n_{t}^{v} \rightarrow n_{t} \text { in } L^{\infty}\left(0, T ; L^{2}\right) \text { weakly star; } \\
& E^{v} \rightarrow E \text { in } L^{2}\left(0, T ; L^{2}\right) \text { strongly and a.e.; } \\
& n^{v} \rightarrow n \text { in } L^{2}\left(0, T ; L^{2}\right) \text { strongly and a.e.; } \\
& n^{v} E^{v} \rightarrow n E \text { in } L^{\infty}\left(0, T ; L^{2}\right) \text { weakly star; } \\
& \left|E^{v}\right|^{2} \rightarrow|E|^{2} \text { in } L^{\infty}\left(0, T ; L^{2}\right) \text { weakly star. }
\end{aligned}
$$

Indeed

$$
\begin{aligned}
\sup _{0 \leq t \leq T}\left\|n^{v} E^{v}-n E\right\| & \leq \sup _{0 \leq t \leq T}\left\|n^{v} E^{v}-n^{v} E\right\|+\sup _{0 \leq t \leq T}\left\|n^{v} E-n E\right\| \\
& \leq\left\|n^{v}\right\|_{L^{\infty}}\left\|E^{v}-E\right\|+\|E\|_{L^{\infty}}\left\|n^{v}-n\right\| \rightarrow 0,
\end{aligned}
$$




$$
\sup _{0 \leq t \leq T}\left\|\left|E^{v}\right|^{2}-|E|^{2}\right\| \leq\left(\left\|E^{v}\right\|_{L^{\infty}}+\|E\|_{L^{\infty}}\right)\left\|E^{v}-E\right\| \rightarrow 0 .
$$

By using the density of $\omega_{j}$ in $L^{2}(\Omega)$, we get the existence of a local generalized solution for the problem (2.1)-(2.6). By continuous extension principle and a prior estimate in Section 2, we can get the existence of the global solution.

\section{Acknowledgements}

This work was supported by the National Natural Science Foundation of China (No. 11271141, No. 11426069 and No.61375006).

\section{References}

[1] Zakharov, V.E. (1972) The Collapse of Langmuir Waves. Soviet Physics_JETP, 35, 908-914.

[2] Bourgain, J. and Colliander, J. (1996) On Well-Posedness of Zakharov System. International Mathematics Research Notices, 11, 515-546. http://dx.doi.org/10.1155/S1073792896000359

[3] Ginibre, J., Tsutsumi, Y. and Velo, G. (1997) On the Cauchy Problem for Zakharov System. Journal of Functional Analysis, 151, 384-436. http://dx.doi.org/10.1006/jfan.1997.3148

[4] Bejenaru, I., Herr, S., Holmer, J. and Tataru, D. (2009) On the 2d Zakharov System with $L^{2}$ Schrodinger Data. Nonlinearity, 22, 1063-1089. http://dx.doi.org/10.1088/0951-7715/22/5/007

[5] Colliander, J., Holmer, J. and Tzirakis, N. (2008) Low Regularity Global Well-Posedness for the Zakharov and KleinGordon-Schrodinger Systems. Transactions of the American Mathematical Society, 9, 4619-4638. http://dx.doi.org/10.1090/S0002-9947-08-04295-5

[6] Pecher, H. (2005) Global Solutions with Infinite Energy for the One-Dimensional Zakharov System. Electronic Journal of Differential Equations, 41, 1-18.

[7] Guo, C.H., Fang, S.M. and Guo, B.L. (2013) Long Time Behavior of the Solutions for the Dissipative Modified Zakharov Equations for Plasmas with a Quantum Correction. Journal of Mathematical Analysis and Applications, 403, 183-192. http://dx.doi.org/10.1016/j.jmaa.2013.01.058

[8] Garcia, L.G., Haas, F., de Oliveira, L.P.L. and Goedert, J. (2005) Modified Zakaharov Equations for Plasmas with a Quantum Correction. Physics of Plasmas, 12. http://dx.doi.org/10.1063/1.1819935

[9] You, S.J., Guo, B.L. and Ning, X.Q. (2012) Initial Boundary Value Problem for Modified Zakharov Equations. Acta Mathematica Scientia, 32B, 1455-1466.

[10] You, S.J., Guo, B.L. and Ning, X.Q. (2012) Initial Boundary Value Problem for Generalized Zakharov Equations. Applied Mathematics, 57, 581-599. http://dx.doi.org/10.1007/s10492-012-0035-6

[11] Guo, B.L., Gan, Z.H. and Zhang, J.J. (2011) Zakharov Equations and Its Solitary Wave Solutions. Science Press. 